\title{
Sobre o gênero Neotropical Tenedos O. Pickard-Cambridge (Araneae: Zodariidae), com a descrição de três espécies novas
}

\author{
David F. Candiani ${ }^{1}$; Alexandre B. Bonaldo ${ }^{1} \&$ Antonio D. Brescovit ${ }^{2}$
}

\begin{abstract}
${ }^{1}$ Museu Paraense Emílio Goeldi. Avenida Perimetral 1901, Terra Firme, 66077-530 Belém, Pará, Brasil. E-mail: dfcandiani@gmail.com; bonaldo@museu-goeldi.br

${ }^{2}$ Laboratório de Artrópodes Peçonhentos, Instituto Butantan. Avenida Vital Brazil 1500, 05503-900 São Paulo, São Paulo, Brasil. E-mail: adbresc@terra.com.br
\end{abstract}

\begin{abstract}
On the neotropical spider genus Tenedos O. Pickard-Cambridge (Araneae: Zodariidae) with the description of three new species. Three new species of Tenedos O. Pickard-Cambridge are described: T. garoa sp. nov., from Parque do Estado, São Paulo, São Paulo, Brazil; T. carlosprestesi sp. nov., from Barra dos Bugres, Mato Grosso, Brazil and T. nancyae sp. nov., from Panguana, Huanuco, Peru. Storena major (Keyserling, 1891) and S. minor (Keyserling, 1891) both known only by juveniles from Blumenau, Santa Catarina, Brazil are transfered to Tenedos and redescribed based on adult females. Storena hirsuta Mello-Leitão, 1941, a male from Rio Negro, Paraná, Brazil, is also transferred to Tenedos and redescribed. New records of Tenedos perfidus Jocqué \& Baert, 2002, T. procreator Jocqué \& Baert, 2002, T. infrarmatus Jocqué \& Baert, 2002 and T. hoeferi Jocqué \& Baert, 2002 from Brazil are presented. Additional documentation of $T$. hoeferi Jocqué \& Baert, 2002 morphology is also presented. Storena petropolitana Mello-Leitão, 1922 from Petrópolis, Rio de Janeiro, Brazil, are transferred here to Epicratinus Jocqué \& Baert, 2005.
\end{abstract}

KEY WORDS. Epicratinus; new combination; new records; taxonomy.

RESUMO. São propostas três espécies novas de Tenedos O. Pickard-Cambridge: $T$. garoa sp. nov., do Parque do Estado, São Paulo, São Paulo, Brasil; T. carlosprestesi sp. nov., de Barra dos Bugres, Mato Grosso, Brasil e T. nancyae sp. nov., de Panguana, Huanuco, Peru. Storena major (Keyserling, 1891) e S. minor (Keyserling, 1891), ambas conhecidas apenas por exemplares imaturos, descritas de Blumenau, Santa Catarina, Brasil são transferidas para Tenedos e redescritas com base em fêmeas adultas. Storena hirsuta Mello-Leitão, 1941, macho de Rio Negro, Paraná, Brasil, é também transferida para Tenedos e redescrita. Novos registros de Tenedos perfidus Jocqué \& Baert, 2002, T. procreator Jocqué \& Baert, 2002, T. infrarmatus Jocqué \& Baert, 2002 e T. hoeferi Jocqué \& Baert, 2002 no Brasil são apresentados. Documentação adicional da morfologia de T. hoeferi Jocqué \& Baert, 2002 é também apresentada. Storena petropolitana Mello-Leitão, 1922, conhecida de Petrópolis, Rio de Janeiro, é transferida para Epicratinus Jocqué \& Baert, 2005.

PALAVRAS-CHAVE. Epicratinus; nova combinação; registros novos; taxonomia.

O gênero Tenedos, proposto por PickaRd-CAMBRidge (1897), foi caracterizado por JocQué (1991), que redescreveu a espécietipo, T. lautus O. Pickard-Cambridge, 1897, procedente da Guatemala, e outras três espécies transferidas para o gênero, duas das quais espécies-tipo dos sinônimos juniores Tijucaia Mello-Leitão, 1940 e Naibena Chamberlin, 1925. Jocqué \& BAERT (2002) incluíram 38 espécies em Tenedos, destacando que a única característica que separa estas espécies das de Ishania Chamberlin, 1925 é o quíleo dividido ou ausente.

Os registros de Tenedos no Brasil são escassos, restritos ao material-tipo das únicas sete espécies conhecidas para o país até o momento: T. brescoviti Jocqué \& Baert, 2002, T. hoeferi Jocqué \& Baert, 2002, T. infrarmatus Jocqué \& Baert, 2002, T. perfidus Jocqué \& Baert, 2002, T. procreator Jocqué \& Baert, 2002, T. reygeli Jocqué \& Baert, 2002 e T. eduardoi (Mello-Leitão, 1925). No presente trabalho são fornecidos registros novos de quatro destas espécies e documentação adicional da morfologia do palpo de T. hoeferi Jocqué \& Baert, 2002, além da proposição de três espécies novas, duas ocorrentes no Brasil e uma no departamento de Huanuco, Peru.

As revisões de Jocqué (1991) e Jocqué \& BAERT (2002) não abordaram as espécies brasileiras descritas em Storena Walckenaer, 1805, gênero que, segundo BAHER \& JocQué (1994), tem distribuição restrita à Austrália. Até o momento, Storena possui quatro espécies válidas para o Brasil. Três destas espécies são aqui transferidas para Tenedos, S. major (Keyserling, 1891) e S. minor

Revista Brasileira de Zoologia 25 (1): 128-138, March, 2008 
(Keyserling, 1891), descritas com base em imaturos de Blumenau, Santa Catarina e aqui redescritas, pela primeira vez, com base em adultos e S. hirsuta Mello-Leitão, 1941, de Rio Negro, Paraná, redescrita com base no holótipo macho. A quarta espécie, $S$. petropolitana Mello-Leitão, 1922, é aqui transferida para Epicratinus, gênero recentemente proposto por JocQué \& BAERT (2005).

\section{MATERIAL E MÉTODOS}

O material examinado é proveniente das seguintes instituições com suas respectivas siglas (curadores entre parênteses): IBSP, Instituto Butantan, São Paulo (A.D. Brescovit); MNRJ, Museu Nacional, Universidade Federal do Rio de Janeiro, Rio de Janeiro (A.B. Kury); MPEG, Museu Paraense Emílio Goeldi, Belém (A. B. Bonaldo); MZSP, Museu de Zoologia da Universidade de São Paulo, São Paulo (R. Pinto da Rocha); SMNK, Staatliches Museum für Naturkunde Karlsruhe, Karlsruhe (H. Höfer).

Todas as medidas estão expressas em milímetros. As descrições seguiram o formato adotado por JocQué \& BAERT (2002), exceto pela inclusão dos diâmetros e das interdistâncias oculares e das medidas de todos os fêmures, patelas, tíbias, tarsos e metatarsos das pernas. A terminologia para as estruturas da genitália seguiu JocQUÉ \& BAERT (2002). Os palpos esquerdos dos machos foram desenhados em posição ventral e retrolateral, considerando-se o posicionamento do címbio independentemente do eventual grau de rotação do bulbo (BonALDo 2000). Uma vez que ambos os espécimes disponíveis de Tenedos carlosprestesi sp. nov. estão com os bulbos expandidos, foi feita uma ilustração adicional do palpo, com o címbio em posição retroventral, para possibilitar a comparação com outros espécimes. O epígino das fêmeas foi estudado imerso em óleo de cravo (Levi 1965), após a digestão dos tecidos moles em pancreatina por 48 horas. As fotomicrografias de varredura foram obtidas no Microscópio Eletrônico de Varredura Zeiss LEO 1450 VP do Laboratório Institucional de Microscopia Eletrônica de Varredura do MPEG.

Abreviaturas utilizadas nas descrições e ilustrações: (AC) abertura de copulação, (AM) apófise média, (ATD) apófise tibial dorsal, (ATR) apófise tibial retrolateral, (ATV) apófise tibial ventral, (d) dorsal, (DC) ducto de copulação, (DF) ducto de fertilização, (E) êmbolo, (Es) espermateca, (Fe) fêmur, (LaA) largura anterior do QOM, (LaP) largura posterior do QOM, (Me) metatarso, (OLA) olho lateral anterior, (OLP) olho lateral posterior, (OMA) olho médio anterior, (OMP) olho médio posterior, (PC) processo cimbial, (p) prolateral, $(\mathrm{Pa})$ patela, (PTB) processo tegular basal, (PTD) processo tegular distal, (QOM) quadrângulo ocular médio, (r) retrolateral, (Ta) tarso, (Ti) tíbia, (Tr) trocanter, (v) ventral.

\section{Tenedos nancyae sp. nov. Figs $1-9$}

Holótipo macho, Rio Yuyapichis, Estación Biológica de Panguana, Panguana, Huanuco, Peru, 21/I-18/II/1984, M. Verhaagh leg. depositado no IBSP 42711. Parátipos: mesmos dados do holótipo, 2 machos (IBSP 42710, 42712), 1 macho (MPEG 02327).
Etimologia. O nome específico é uma homenagem a aracnóloga Nancy França Lo Man Hung, esposa do primeiro autor.

Diagnose. Machos assemelham-se aos de T. peckorum (ver JoCQUÉ \& BAERT 2002, figs 29 a, b) pela ATR larga e AM longa e bífida, mas difere desta espécie pela $\mathrm{AM}$ com área distal não tão alongada e PTD mais largo (Figs 1, 4, 8).

Descrição. Macho (holótipo): comprimento total 3,92; carapaça, comprimento 2,11; largura 1,43. Fila de olhos anterior ligeiramente reta, fila posterior procurva; carapaça e quelíceras marrom-alaranjadas; esterno laranja-claro; pernas laranja-amareladas; fêmures marrom-claros; abdômen cinzaescuro com cinco manchas brancas, duas anteriores, duas medianas e uma posterior (Fig. 7).

Olhos. Diâmetros: OMP 0,11, OLP 0,10, OLA 0,11, OMA 0,05; QOM: comprimento 0,28, LaA 0,15, LaP 0,24; Interdistâncias: OMP-OMP 0,04, OLP-OMP 0,07, OMA-OMA 0,01, OLA-OMA 0,02, OLA-OLP 0,04, OMA-OMP 0,10. Pernas: I: Fe 1,23/Pa 0,42/Ti 1,15/ Me 1,11/Ta 0,73/total 4,64. II: 1,08/0,42/0,88/0,88/0,61/3,87. III: 1,04/0,42/0,76/1,0/0,61/3,83. IV: 1,27/0,53/0,88/1,53/0,61/4,82. Fórmula 4123. Espinulação: I: Fe v0, d1-1-1, p0-0-1, r0; Pa v0, d0, p0, r0; Ti v1-1-2, d0, p1-0-0, r0-1-0; Me v1-0-2, d0, p0-0-1, r0-0-1. II: Fe v0, d1-1-1, p0, r0; Pa v0, d0, p0, r0; Ti v1-1-2, d0, p0, r0; Me v1-0-2, d0, p0-1-1, r0. III: Fe v0, d0-1-1, p0, r0; Pa v0, d0-0-1, p0-10, r1-1-0; Ti v2-1-2, d1-1-1, p0-1-1, r1-0-1; Me v0-2-2, d0-1-2, p0-12, r0-1-1. IV: Fe v0, d1-1-1, p0, r0; Pa v0, d0-0-1, p0-1-0, r1-1-0; Ti v1-1-2, d1-1-1, p2-0-1, r1-0-1; Me v0-1-2-2, d1-1-2, p2-1-2, r1-1-2.

Palpo. ATR robusta, quilhada, com extremidade afilada em forma de cunha (Fig. 9). ATV curta, romba, em forma de colher (Figs 8 e 9). ATD ausente. Tégulo pequeno e esclerotizado. Címbio com dois espinhos apicais (Figs 8 e 9). PTD muito desenvolvido, de ponta muito fina (Figs 1, 2, 4 e 8). Êmbolo com superfície porosa, base engrossada, e ápice não projetado, (Figs 3, 5 e 6). AM com base membranácea, longa e ápice em forma de taça, esclerotizada, bífida na região distal, com extremidade posterior triangular (Fig. 8).

Fêmea. Desconhecida.

Variação. Comprimento: (10 machos) total 3,34-4,0, carapaça 1,73-2,0, FeI 1,07-1,26. A coloração da carapaça varia de laranja-escuro a vermelho-alaranjado; AM pode estar retraída (Fig. 1) ou distendida (Fig. 8); borda retrolateral da base do PTD pode ser esclerotizada.

Distribuição. Conhecida somente na localidade-tipo.

Material adicional. Peru, Huanuco: Rio Yuyapichis (Estación Biológica de Panguana), 2 machos, 21/I-18/II/1984, M. Verhaagh leg. (IBSP 42709, 42713).

\section{Tenedos garoa sp. nov.}

Figs $10-25$ e 30

Holótipo macho, BrasiL, São Paulo: São Paulo (Parque do Estado), 1-7/XI/2001, J.R. Valvassori leg. depositado no IBSP 44465. Parátipos: 2 machos, 1 fêmea (IBSP 44598) e 2 machos, 2 fêmeas (MPEG 02328, 02329), com mesmos dados do holótipo.

Revista Brasileira de Zoologia 25 (1): 128-138, March, 2008 

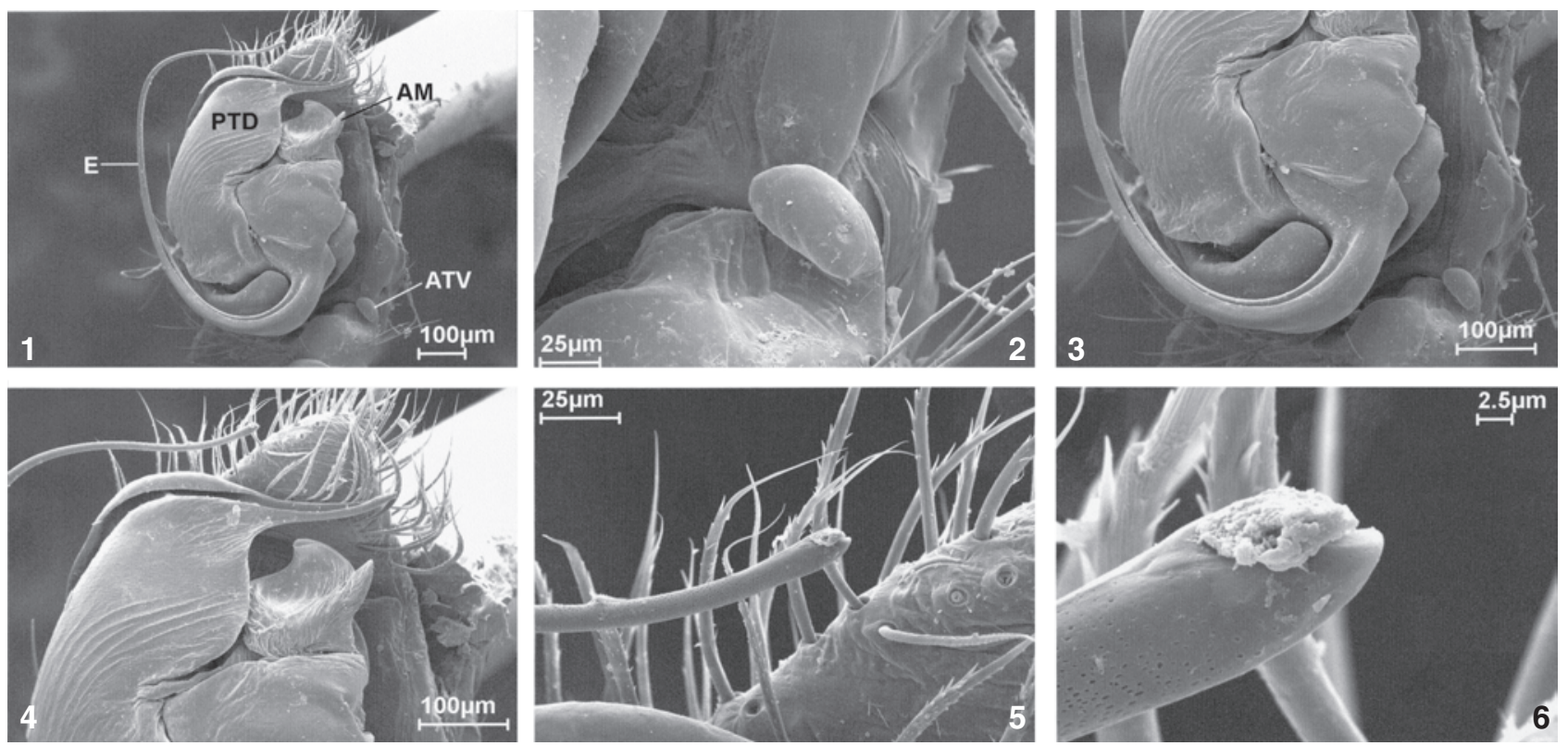

Figuras 1-6. Tenedos nancyae sp. nov., palpo do macho, retroventral: (1) bulbo; (2) apófise tibial ventral; (3) base do bulbo; (4) ápice do bulbo; (5-6) ápice do êmbolo. (AM) Apófise média, (ATV) apófise tibial ventral, (E) êmbolo, (PTD) processo tegular distal.

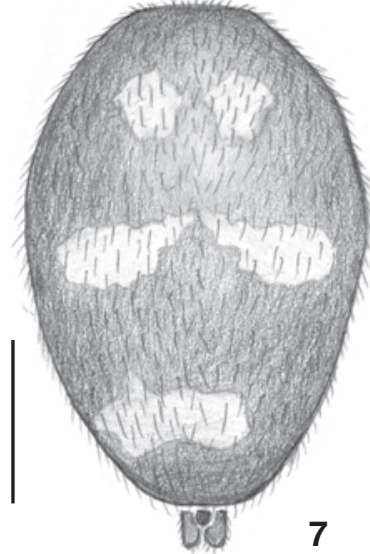

7
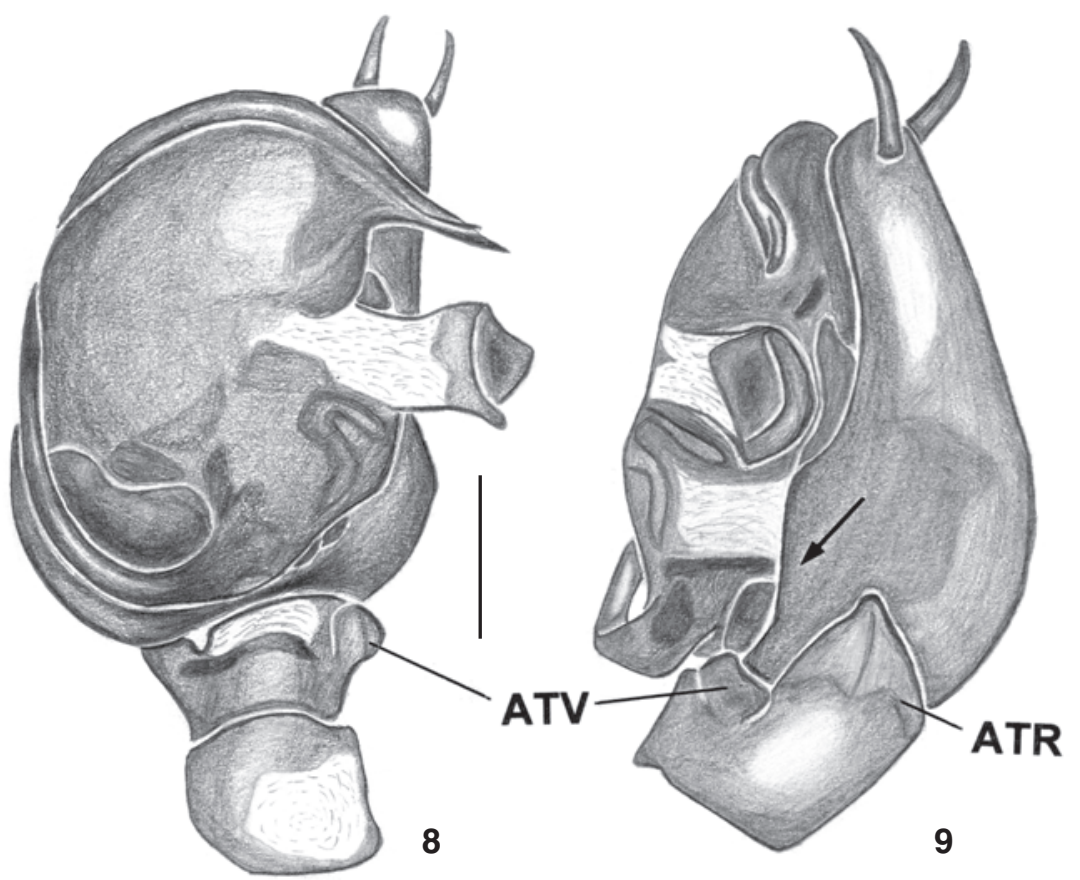

Figuras 7-9. Tenedos nancyae sp. nov., abdômen e palpo do macho: (7) abdômen, dorsal; (8) palpo do macho, ventral; (9) idem, retrolateral. (ATV) Apófise tibial ventral, (ATR) apófise tibial retrolateral. Seta: processo címbial. Barras: Fig. 7, 0,5 mm; Figs 8, 9, 0,25 mm.

Etimologia. O nome específico refere-se à chuva fina e persistente, característica do inverno na capital paulista. A palavra garoa surgiu a partir da palavra espanhola garúa, que por sua vez surgiu a partir da palavra caruja, de origem portuguesa, que significa, em seu sentido original, orvalho.

Diagnose. Machos de Tenedos garoa sp. nov. assemelhamse aos de T. eduardoi (ver JocQué \& BAERT 2002: figs 13 a-b) pela presença de três apófises tibiais, PTD grande, de implantação

Revista Brasileira de Zoologia 25 (1): 128-138, March, 2008 

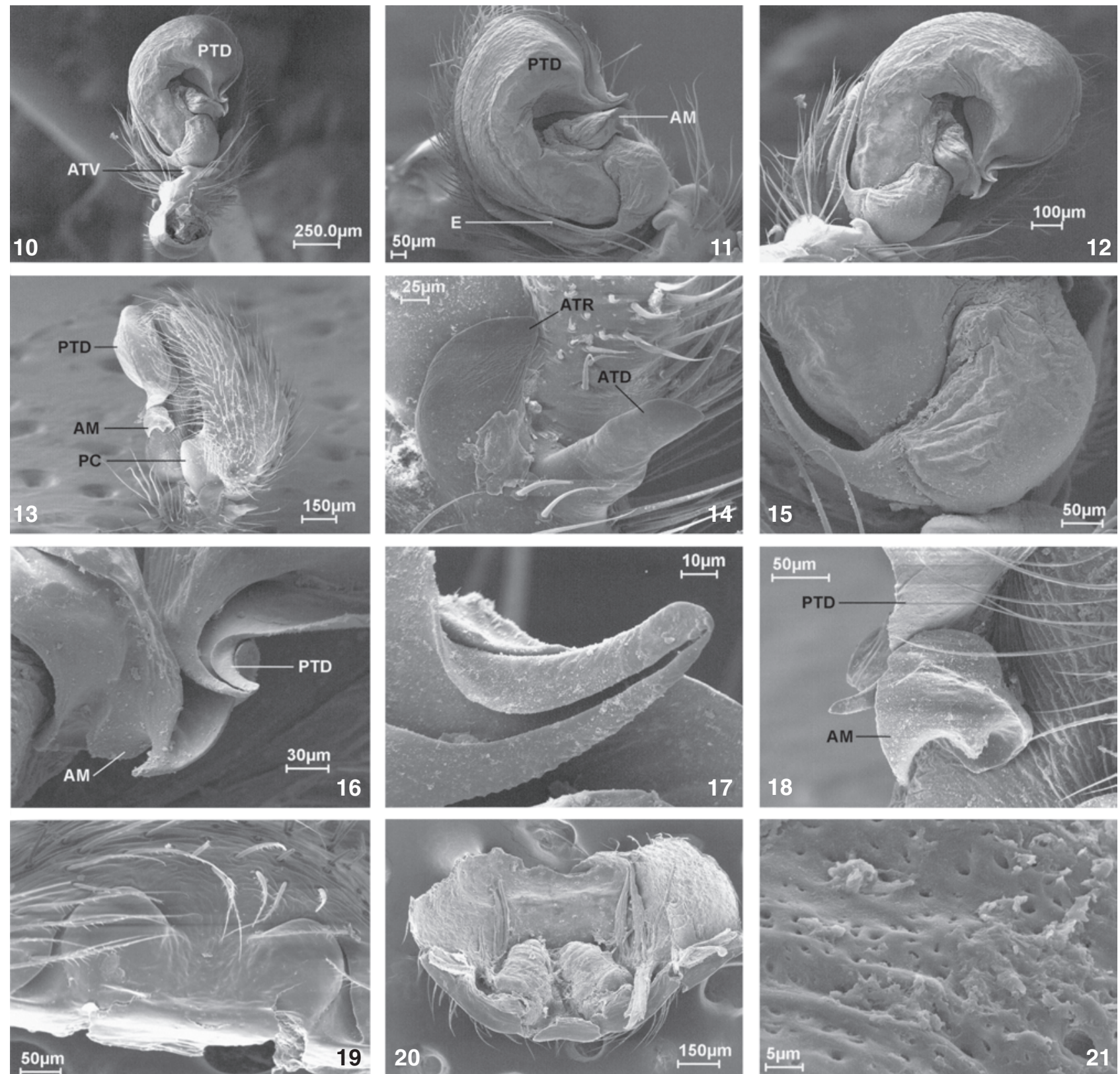

Figuras 10-21. Tenedos garoa sp. nov., palpo do macho e epígino da fêmea: (10-12) palpo do macho, ventral; (13) idem, retrolateral; (14) ápice da tíbia, retrolateral; (15) base do êmbolo, ventral; (16) ápice da apófise média e processo tegular distal, ventral; (17) ápice do processo tegular distal, ventral; (18) ápice da apófise média e processo tegular distal, retrolateral; (19) epígino, ventral; (20) epígino dorsal; (21) superfície da espermateca, dorsal. (E) Êmbolo, (AM) apófise média, (ATV) apófise tibial ventral, (ATR) apófise tibial retrolateral, (ATD) apófise tibial dorsal, (PC) processo cimbial, (PTD) processo tegular distal.

mediana, levemente deslocada para a região prolateral do palpo, formando, em toda sua extensão, uma calha para acomodar o êmbolo. Diferem desta espécie pela ATR laminar de ápice rombo e ATD de ápice rombo (Figs 14 e 23); AM em forma de taça, com uma profunda calha mediana que acolhe o ápice do PTD no bulbo não expandido (Figs 16, 18, 22 e 23). As fêmeas de $T$. garoa sp. nov. assemelham-se as de T. figaro (ver JOCQUÉ \& BAERT 2002: figs 16 c, e) pela presença de ductos de copulação helicoidais; mas diferem desta espécie pelos ductos de copulação longos, espiralados, conspícuos por transparência e pela au- 
sência de escapo epiginal (Figs 19, 24 e 25).

Descrição. Macho holótipo: comprimento total 5,69; carapaça, comprimento 2,92, largura 2,05. Carapaça marrom-escura; quelíceras marrom-escuras; esterno marrom-alaranjado, porção central mais clara que as bordas, com pêlos em toda sua extensão; pernas laranja-escuras, com coxas amarelas, Fe e Pa marrons, Ti I amarelo-claro, Ti III e IV, Me e Ta marrom-escuro; abdômen cinza com manchas brancas: dorso com uma mancha irregular anterior mediana e manchas esparsas na região posterior (Fig. 30).

Olhos. Diâmetros: OMP 0,15, OLP 0,13, OLA 0,13, OMA 0,07; QOM: comprimento 0,28, LaA 0,23, LaP 0,27; Interdistâncias: OMP-OMP 0,04, OLP-OMP 0,10, OMA-OMA 0,04, OLA-OMA 0,05, OLA-OLP 0,05, OMA-OMP 0,06. Pernas: I: Fe 1,88/Pa 0,76/Ti 1,8/ Me 1,5/Ta 1,07/total 7,01. II: 1,59/0,76/1,15/1,5/0,96/5,96. III: 1,63/ 0,76/1,15/1,5/0,92/5,96. IV: 2,0/0,76/1,65/2,0/1,26/7,67. Fórmula 4123. Espinulação: I: Me v2-1-2, d0, p0-0-1, r0-0-1. Ti v2-2-2, d0, p0, r0; Pa v0, d0, p0, r0; Fe v0, d1-1-1, p0-0-1, r0. II: Me v2-12, d0, p0-0-1, r0. Ti v1-2-2, d0, p0-1-0, r0; Pa v0, d0, p0, r0; Fe v0, d1-1-1, p0, r0. III: Me v2-2-2, d0-1-1, p1-1-1, r1-1-2. Ti v2-2-2, d11-1, p0-1-1, r2-0-1; Pa v0, d0-1-1, p0-1-0, r2-1-0; Fe v0, d1-1-3, p0, r0. IV: Me v2-2-2, d0-1-2, p1-1-2, r1-1-2. Ti v2-2-2, d1-1-1-1, p0-11, r1-1-1-1; Pa v0, d0-1-1, p0-1-0, r2-1-0; Fe v0, d1-1-1, p0-0-1, r0.

Palpo. Tíbia com três apófises: ATV pequena, simples e inconspícua com ápice direcionado prolateralmente; ATR simples e laminar; ATD comprida, larga e romba (Figs 13, 14 e 23). Címbio com cinco espinhos apicais, distribuídos de forma irregular (Figs 22 e 23); processo cimbial conspícuo, expandido retrolateralmente (Figs 22 e 23). PTD grande, membranáceo na base, inserido na região mediana do tégulo, orientado retrolateralmente (Figs 10-12); margem retrolateral e ápice do PTD fortemente esclerotizadas, ápice curvo, direcionado retrolateralmente formando uma calha que acolhe o ápice do êmbolo no bulbo não expandido (Figs 16-18 e 22). Ponta da AM formando uma calha que acomoda a ponta do PTD (Fig. 16-18). Êmbolo longo, inserido na porção prolateral basal do tégulo (Fig. 15 e 22).

Fêmea (Parátipo, IBSP 44598). Comprimento total 6,61; carapaça, comprimento 2,92; largura 2,6. Coloração como no macho.

Olhos. Diâmetros: OMP 0,07, OLP 0,05, OLA 0,10, OMA 0,05; QOM: comprimento 0,34, LaA 0,21, LaP 0,26; Interdistâncias: OMP-OMP 0,04, OLP-OMP 0,08, OMA-OMA 0,04, OLA-OMA 0,05, OLA-OLP 0,05, OMA-OMP 0,07. Pernas: I: Fe 1,84/Pa 0,64/Ti 1,52/Me 1,11/Ta 0,94/total 6,05. II: 1,46/ 0,76/1,35/1,05/0,88/5,5. III: 1,62/0,76/1,17/1,23/0,88/5,66. IV: 1,96/0,76/1,70/1,88/1,23/7,53. Espinulação: I: Me v1-0-2, d0, p0, r0; Ti v1-0-0, d0, p0, r0; Fe v0, d1-1-1, p0, r0; Pa v0, d0, p0, r0. II: Me v1-0-2, d0, p0-0-1, r0; Ti v1-1-0, d0, p0, r0; Pa v0, d0, p0, r0; Fe v0, d1-1-1, p0, r0. III: Me v2-1-2, d2-3-2, p0-0-2, r1-12; Ti v2-2-2, d1-1-1, p0-0-1, r2-0-1; Pa v0, d0-1-0, p0-1-0, r1-11; Fe v0, d1-0-1, p0, r0. IV: Me v2-1-2, d2-1-2, p1-1-2, r1-1-2; Ti v2-2-2, d1-1-1-1, p1-1-1, r2-0-1; Pa v0, d0-1-2, p0-1-0, r1-1-1;
Fe v0, d1-1-1, p0, r0.

Epígino. Placa epiginal com átrio delimitado por sulcos latero-anteriores, procurvos, oblíquos, onde se localizam as AC (Figs 19 e 24). DC longos, espiralados, conspícuos por transparência na face ventral do epígino; DF pequenos, inseridos na região posterior; espermatecas globulares, com superfície porosa (Figs 20, 21 e 25).

Variação. Comprimento: (10 machos) total 5,58-6,29, carapaça 2,88-3,17, FeI 1,70-2,0, (4 fêmeas) total 6,35-8,0, carapaça 2,76-3,17, FeI 1,41-1,76. A coloração da carapaça varia de marrom-amarelado a marrom-avermelhado; pernas de amarelo a amarelo-esverdeadas. No palpo do macho, o PTD varia de membranáceo a esclerotinizado.

Distribuição. Conhecida somente na localidade-tipo.

Material adicional. Brasil, São Paulo: São Paulo (Parque do Estado), 32 machos, 2 fêmeas, 1-7/XI/2001, J.R. Valvassori leg. (IBSP 44589-44597; 44466-44469; 44462-44464; 44600; $44580 ; 44470)$.

\section{Tenedos major (Keyserling, 1891) comb. nov.} Figs 26, 27, 31

Habronestes major Keyserling, 1891: 158, pr. 4, fig. 109 (Holótipo fêmea imatura, Blumenau, Santa Catarina, Brasil, depositado no "The Natural History Museum", Londres, $\mathrm{n}^{\circ}$ 1890.7.1.8410 (1890/2893), examinado por A.A. Lise).

Storena major: Petrunkevitch (1911: 154); Mello-Leitão (1927: 398); Platnick (2006).

Nota. Adultos desta espécie, procedentes da localidadetipo, foram reconhecidos pelo padrão de coloração do abdômen e pelo tamanho dos espécimes, maiores do que em T. minor (Keyserling, 1891).

Diagnose. Fêmeas de Tenedos major (Keyserling, 1891) assemelham-se as de T. minor (Keyserling, 1891) (ver Figs 28 e 29) pelas aberturas de copulação localizadas na região anterior do epígino, mas diferem pelo porte avantajado e epígino com AC sob sulcos sub-retos, longos, delimitando um par de elevações sub-circulares posteriores (Fig. 26).

Descrição. Macho. Desconhecido

Fêmea (IBSP 53887): comprimento total 8,19; carapaça, comprimento 3,96; largura 2,42. Carapaça marrom-escuro; quelíceras marrom-avermelhadas; enditos amarelos e lábio marrom-avermelhado; esterno laranja-avermelhado; pernas amarelo-esverdeadas, com coxas amarelas; abdômen negroacinzentado, com manchas irregulares na região anterior, um par de manchas medianas, três manchas consecutivas em forma de "V" invertido, uma retangular na porção posterior e uma pequena, transversal, logo abaixo (Fig. 31).

Olhos. Diâmetros: OMP 0,15, OLP 0,14, OLA 0,14, OMA 0,11; QOM: comprimento 0,31, LaA 0,26, LaP 0,34; Interdistâncias: OMP-OMP 0,05, OLP-OMP 0,20, OMA-OMA 0,05, OLA-OMA 0,11, OLA-OLP 0,10, OMA-OMP 0,13. Pernas: I: 

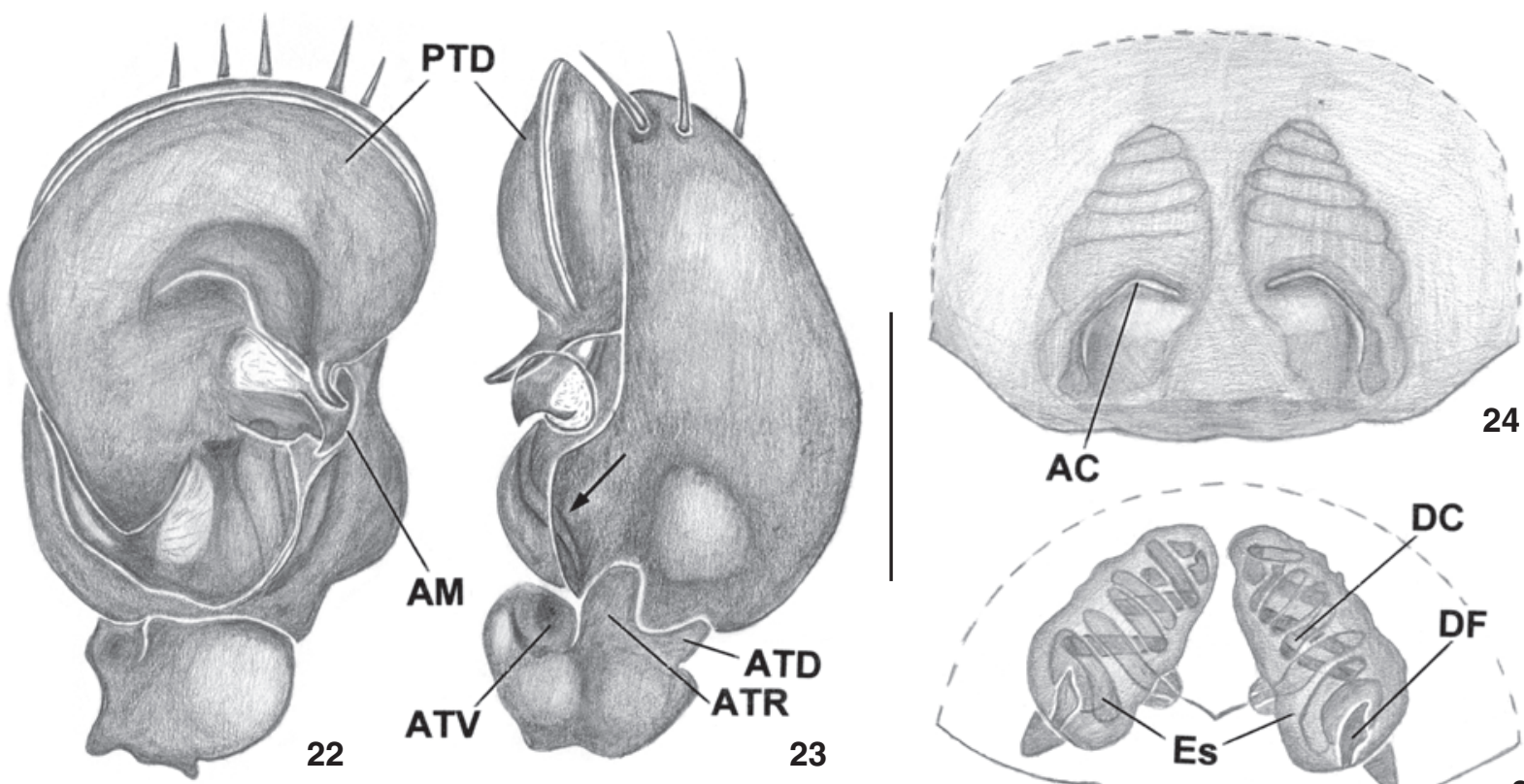

24
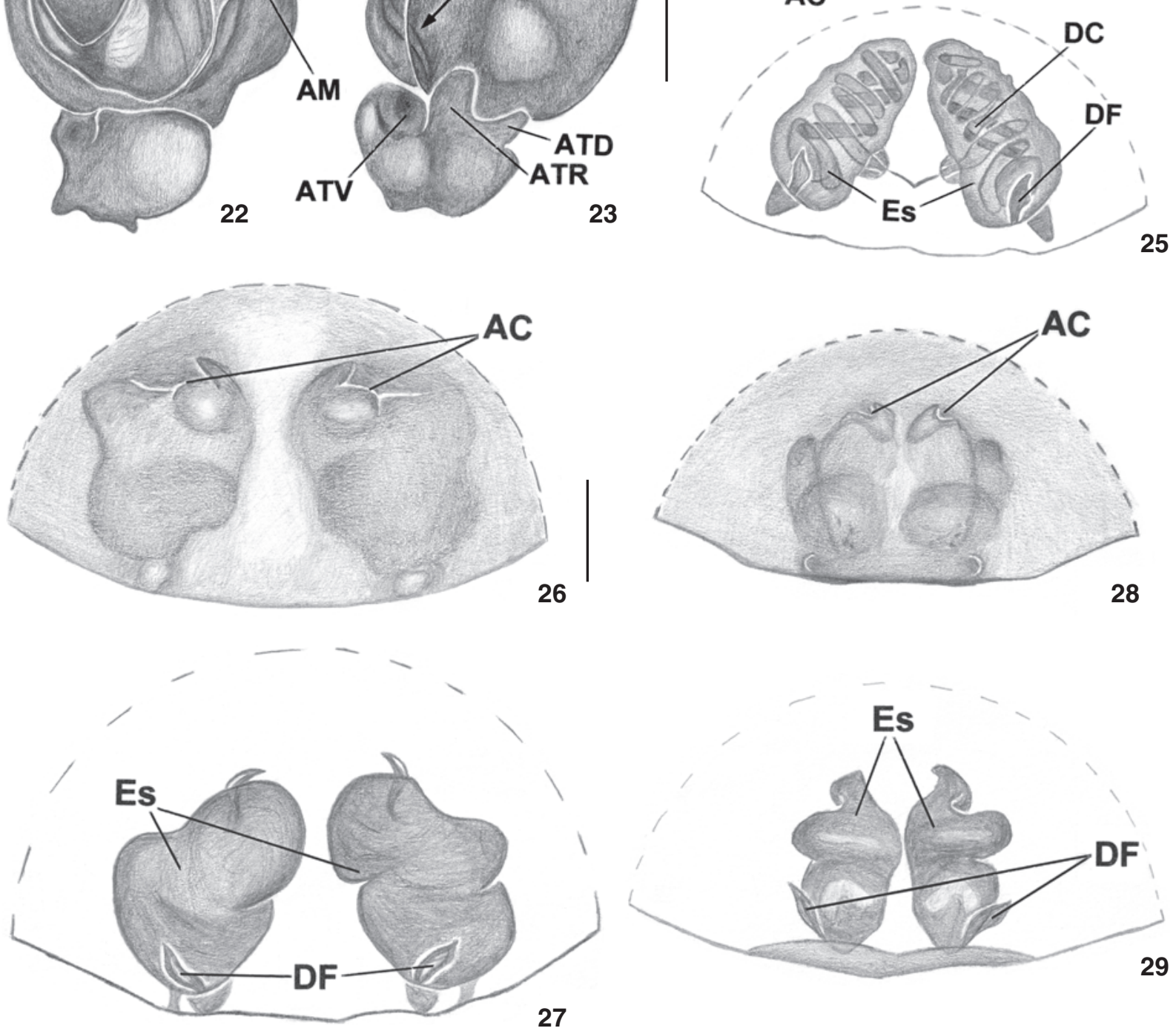

28

Figuras 22-29. (22-25) Tenedos garoa sp. nov., palpo do macho e epígino: (22) palpo, ventral; (23) idem, retrolatreral; (24) epígino, ventral; (25) idem, dorsal. (AM) Apófise média, (ATV) apófise tibial ventral, (ATR) apófise tibial retrolateral, (ATD) apófise tibial dorsal, (PTD) processo tegular distal, (AC) abertura copulatória, (Es) espermatecas, (DF) ducto de fertilização, (DC) ducto de copulação. Seta: processo cimbial. Barras: 1,0 mm. (26-29) Epíginos: (26-27) T. major comb. nov., (26) ventral; (27) dorsal; (28-29) T. minor comb. nov., (28) ventral, (29) dorsal. (AC) Abertura copulatória, (Es) espermatecas, (DF) ducto de fertilização. Barra: 0,25 mm. 
Fe 2,38/Pa 0,61/Ti 1,26/Me 0,96/Ta 0,84/total 6,05. II: 2,19/0,61/ 1,03/0,96/0,76/5,55. III: 1,92/0,57/0,84/0,92/0,80/5,05. IV: $2,42 /$ $0,61 / 1,38 / 1,42 / 0,84 / 6.67$. Fórmula 4123. Espinulação: I: Fe v0, d1-1-1, p0, r0; Pa v0, d0, p0, r0; Ti v1-1-1, d0, p0-1-0, r0; Me v22-2, d0, p0, r0. II: Fe v0, d1-1-1, p0, r0; Pa v0, d0, p0, r0; Ti v1-11-2, d0, p0-1-0, r0; Me v2-1-2-2, d0, p0-0-2, r0. III: Fe v0, d1-2-2, p0, r0; Pa v0, d0-1-1-1, p0-1-0, r2-2-1; Ti v2-2-2, d2-1-1, p1-1-1, r1-2-1-1; Me v1-2-2-2, d1-1-2-2, p1-1-1-2, r1-1-2. IV: Fe v0, d1-12, p0, r0; Pa v0, d0-1-1-1, p0-1-0, r2-2-1; Ti v2-2-1-2, d2-1-1-1, p1-1-0-1, r2-2-1-2; Me v2-2-2-2, d2-1-2-2, p1-0-1-2, r2-1-1-1-2.

Epígino. Átrio não delimitado, AC localizadas sob sulcos transversais anteriores, superfície do epígino com um par de elevações. Internamente, espermatecas com projeções laterais inconspícuas, anteparo dos DF representado por um par de pequenas placas posteriores esclerotinizadas (Fig. 27).

Distribuição. Conhecida somente na localidade-tipo.

Material examinado. Brasil, Santa Catarina: Blumenau (Parque Municipal das Nascentes do Ribeirão Garcia), 2 fêmeas, 21-28/I/2003, Equipe BIOTA leg. (IBSP 53887 e 53888).

\section{Tenedos minor (Keyserling, 1891) comb. nov.}

\section{Figs $28,29,32$}

Habronestes minor Keyserling, 1891: 158, pr. 4, fig. 109 (Holótipo fêmea imatura, Blumenau, Santa Catarina, Brasil, depositado no "The Natural History Museum", Londres, $\mathrm{n}^{\circ}$ 1890.7.1.8411 (1890/2894), examinado por A.A. Lise).

Storena minor: Petrunkevitch (1911: 154); Mello-Leitão (1927: 398); Platnick (2006).

Nota. Adultos desta espécie, procedentes da localidadetipo, foram reconhecidos pelo padrão de coloração do abdômen e pelo tamanho dos espécimes, menores do que em T. major.

Diagnose. Fêmeas de Tenedos minor (Keyserling, 1891) assemelham-se as de T. major (Keyserling, 1891) (ver Figs 27 e 28) pelas aberturas de copulação localizadas na região anterior do epígino; diferem pelo menor porte e pelo epígino com AC inseridos em sulcos curtos, procurvos; superfície do epígino sem elevações (Figs 28 e 29).

Descrição. Macho. Desconhecido.

Fêmea: comprimento total 5,26; carapaça, comprimento 2,57; largura 1,57. Carapaça marrom-escura; quelíceras amarelo-claras; esterno marrom-esverdeado com bordas vermelhoalaranjadas; pernas verde-amareladas; abdômen negroacinzentado, mancha anterior em forma de ferradura partida ao meio e duas manchas menores imediatamente abaixo, duas manchas na região mediana e três manchas em forma de "V" invertido logo abaixo (Fig. 32). Pernas com grupos de cerdas ventro-distais em todos os metatarsos.

Olhos. Diâmetros: OMP 0,11, OLP 0,11, OLA 0,13, OMA 0,08; QOM: comprimento 0,28, LaA 0,17, LaP 0,23; Interdistâncias: OMP-OMP 0,04, OLP-OMP 0,13, OMA-OMA 0,05, OLA-OMA 0,10, OLA-OLP 0,05, OMA-OMP 0,08. Pernas. I: Fe 1,44/Pa 0,61/Ti 1,26/Me 0,96/Ta 0,76/total 5,03. II: 1,26/ 0,61/0,61/0,96/0,69/4,13. III: 1,14/0,57/0,76/0,96/0,76/4,19. IV:
1,37/0,57/1,15/1,53/0,96/5,58. Fórmula 4132. Espinulação. I: Fe v0, d1-1-1, p0, r0; Pa v0, d0, p0, r0; Ti v1-0-2, d0, p0-1-0, r0; Me v0-1-2, d0, p0-0-1, r0. II: Fe v0, d1-1-1, p0, r0; Pa v0, d0, p0, r0; Ti v1-1-1-2, d0, p0-1-0, r0; Me v1-1-2, d0, p0, r0. III: Fe v0, d1-0-1, p0, r0-1-0; Pa v0, d0-1-0, p0-1-0, r2-2-0; Ti v2-2-2, d11-1, p0-1-1, r1-1-1-1; Me v2-0-2, d0-1-2-2, p1-1-1, r2-1-1. IV: Fe v0, d1-1-1, p0, r0; Pa v0, d0-1-1-1, p0-1-0, r2-2-1; Ti v1-1-2, d11-1-1, p1-1-1, r1-1-1-1; Me v2-1-2, d0-1-1-2, p1-1-1, r1-1-1.

Epígino. Placa epiginal sem átrio delimitado; internamente com AC localizadas em sulcos anteriores curtos, procurvos, não delimitando elevações posteriores. Projeções laterais dos DC conspícuas, anteparo dos DF representado por uma única placa posterior esclerotizada (Fig. 29).

Variação. Comprimento: (7 fêmeas) total 4,65-5,8, carapaça 2,15-2,65, FeI 1,20-1,45. Algumas fêmeas apresentam uma quilha longitudinal esclerotizada na superfície ventral do epígino, sobre cada espermateca.

Distribuição. Conhecido somente para a localidade-tipo.

Material examinado. Brasil, Santa Catarina: Blumenau (Parque Municipal Nascentes do Ribeirão Garcia), 6 fêmeas, 21-28/I/ 2003, Equipe BIOTA leg. (IBSP 53886, 53889-53891, 5389353894).

\section{Tenedos hirsutus (Mello-Leitão, 1941) comb. nov. Figs 33-35}

Storena hirsuta Mello-Leitão, 1941: 248 (Holótipo macho, Rio Negro, Paraná, Brasil, depositado no MNRJ 58285, examinado); Platnick (2006).

Diagnose. Machos assemelham-se aos de T. carlosprestesi sp. nov. (Figs 37-39) por compartilharem PTB em forma de gancho; diferem destes pela AM esclerotizada, acomodando o PTD quando em repouso; PTB de base alargada e ápice agudo, êmbolo curto, delgado e flexível, não encaixado no PTD (Fig. 34).

Descrição. Macho holótipo: comprimento total 5,75; carapaça, comprimento 2,65; largura 1,5. Carapaça marromavermelhado; quelíceras amarelo-claro; esterno marrom-amarelado; pernas amarelo-claras com coxas e fêmures de coloração marrom-avermelhado; abdômen cinza, apresentando um par de manchas arredondadas anteriores, uma mancha triangular média, uma faixa transversal mediana e duas manchas transversais na porção posterior (Fig. 33).

Olhos. Diâmetros: OMP 0,08, OLP 0,07, OLA 0,08, OMA 0,05. QOM: comprimento 0,20, LaA 0,15, LaP 0,23. Interdistâncias: OMP-OMP 0,05, OLP-OMP 0,05, OMA-OMA 0,04, OLA-OMA 0,04, OLA-OLP 0,04, OMA-OMP 0,05. Pernas. I: Fe 1,3/Pa 0,57/Ti 1,15/Me 1,19/Ta 0,73/total 4,94. II: 1,15/0,46/1,15/ 1,07/0,69/4,52. III: 1,08/0,53/0,92/1,15/0,76/4,44. IV: 1,44/0,53/ 1,23/1,53/0,80/5.53. Fórmula 4123. Espinulação. I: Fe v0, d0-10-1-1, p0, r0; Pa v0, d0, p0, r0; Ti v2-2-2-2, d0, p0, r0; Me v2-0-2, d0, p0, r0. II: Fe v0, d0-1-0-2-1, p0, r0; Pa v0, d0, p0, r0; Ti v1-10, d0, p1-0-0, r0; Me v2-2-2, d0, p0, r0. III: Fe v0, d2-0-2-2, p0, r0; Pa v0, d0-1-2, p0-1-0, r2-0-0; Ti v2-2-2, d1-1-0-0, p0-1-1-1, r1-1-1; Me v2-0-1-2, d2-2-0-2, p1-0-1-2, r1-1-2. IV: Fe v0, d0-1-0-

Revista Brasileira de Zoologia 25 (1): 128-138, March, 2008 

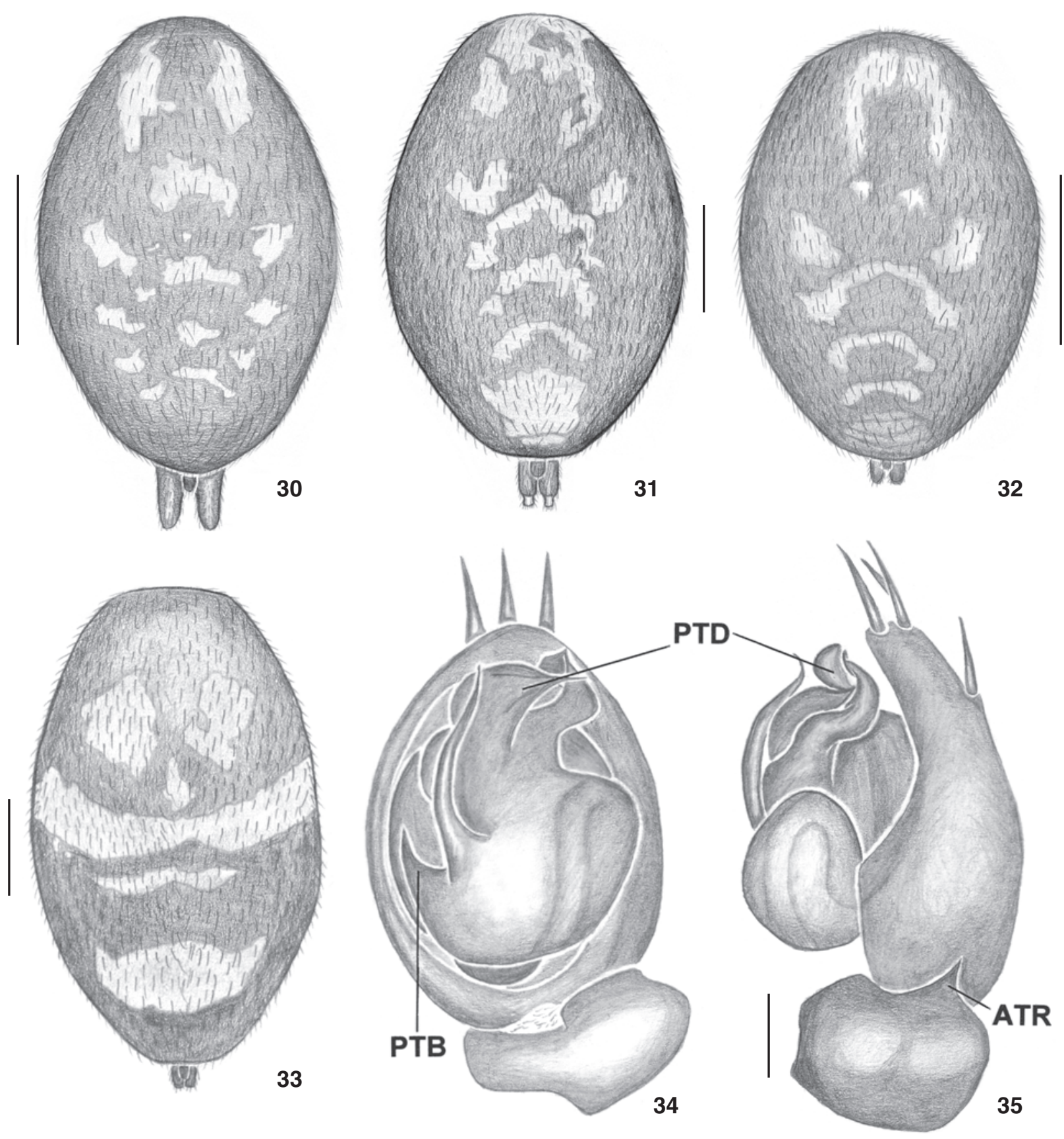

Figuras 30-35. (30-32) Abdômen, dorsal: (30) T. garoa sp. nov., macho; (31) T. major comb. nov., fêmea; (32) T. minor comb. nov., fêmea; (33-35) Tenedos hirsutus comb. nov., abdômen e palpo do macho: (33) abdômen dorsal; (34) palpo, ventral; (35) idem, retrolateral. (ATR) Apófise tibial retrolateral, (PTB) processo tegular basal, (PTD) processo tegular distal. Barras: Figs 30-32 = 1,0 mm, Fig. $33=$ $0,5 \mathrm{~mm}$; Figs $34,35=0,25 \mathrm{~mm}$.

1-2, p0, r0; Pa v0, d0-1-2, p1-1-0, r1-1-1; Ti v2-3-2, d1-0-1-1, p11-1, r0-1-2-1; Me v2-0-2-2, d2-2-2, p2-2-2, r1-1-0-2.

Palpo. ATR única, curta, de base larga e ponta fina (Fig. 34); címbio com três espinhos apicais e um dorsal (Figs 34 e
35); tégulo mais largo que longo. PTD estreito, de base fina, sem função de calha para acomodar o êmbolo. AM esclerotizada. PTB prolateral, de ápice agudo, próximo a base do êmbolo. Êmbolo curto, rígido (Fig. 34). 
Fêmea. Desconhecida.

Distribuição. Conhecida somente para a localidade-tipo. Material examinado. Somente o material-tipo.

\section{Tenedos carlosprestesi sp. nov.}

Figs 36-39

Holótipo macho, Brasil, Mato Grosso: Barra dos Bugres, XI/1983, A. Cerrutti leg. depositado no IBSP 59109. Parátipo. 1 macho, com mesmos dados do holótipo (MPEG 02330).

Etimologia. O nome específico é uma homenagem a Luis Carlos Prestes, líder da Coluna Prestes, que percorreu aproximadamente 25 mil quilômetros pelo interior do Brasil, desde o Rio Grande do Sul até o nordeste brasileiro, passando pelo Mato Grosso, entre outubro de 1924 e fevereiro de 1927.

Diagnose. Machos de Tenedos carlosprestesi sp. nov. assemelham-se aos de T. hirsutus (Figs 34 e 35) pela presença de PTB em forma de gancho; diferem destes pela AM hialina; PTB virguliforme, curto, de ápice rombo (Figs 37 e 38); êmbolo longo, encaixado no PTD a partir da região mediana (Figs 37-39).

Descrição. Macho (holótipo): comprimento total 3,88; carapaça, comprimento 1,81; largura 1,03. Carapaça marrom e quelíceras marrom; esterno marrom, esterno com porção central mais clara que as bordas; pernas marrom-alaranjadas, fêmures e coxas marrom-claro; abdômen cinza escuro com quatro manchas brancas: duas anteriores e duas medianas, as posteriores, cada uma, com cinco pequenas manchas mais claras (Fig. 36). Carapaça reticulada apresentando poucos pêlos próximos aos olhos. Pêlo longo único implantado logo abaixo dos OMA.

Olhos. Diâmetros: OMP 0,08, OLP 0,1, OLA 0,08, OMA 0,05.QOM: comprimento 0,13, LaA 0,14, LaP 0,18; Interdistâncias: OMP-OMP 0,04, OLP-OMP 0,07, OMA-OMA 0,11, OLA-OMA 0,02, OLA-OLP 0,02, OMA-OMP 0,04. Pernas.

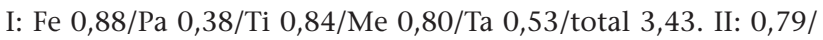
0,38/0,84/0,76/0,53/3,3. III: 0,72/0,57/0,57/0,69/0,46/3,01. IV: $0,92 / 0,42 / 0,80 / 0,92 / 0,46 / 3,52$. Fórmula 4123. Espinulação. I: Fe v0, d1-1-0, p0, r0; Pa v0, d0, p0, r0; Ti v0, d0, p1-0-0, r0; Me v2-0-2, d0, p1-0-1, r0. II: Fe v0, d1-1-1, p0, r0; Pa v0, d0, p0, r0; Ti v1-0-0, d1-0-0, p1-0-0, r0-0-0; Me v1-0-2, d0, p0-0-1, r0. III: Fe v0, d0-2-2, p0, r0; Pa v0, d0-0-1, p0-1-0, r1-1-0; Ti v0-1-2, d1-1-0, p1-0-1, r1-0-1; Me v1-0-3, d1-1-2, p0-1-2, r1-0-1. IV: Fe v0, d1-1-2, p0, r0; Pa v0, d0-0-1, p0-0-1, r2-1-0; Ti v1-1-1, d1-10, p1-0-1, r1-0-1; Me v1-1-3, d1-2-0, p0-0-2, r0-1-1.

Palpo. ATR estreita, curta de ponta fina (Fig. 38); címbio com dois espinhos apicais (quebrados no holótipo e no parátipo); PTD grande, robusto, de base larga e ponta fina, acomodando a ponta do êmbolo em uma calha no palpo não expandido (Figs 37 e 39). AM membranácea com ponta bífida levemente esclerotizada (Figs 37-39). PTB prolateral, virguliforme, próximo à base do êmbolo (Fig. 38). Êmbolo de base larga, comprido e flexível, originando-se na porção proximal-prolateral do tégulo (Fig. 37).

Fêmea. Desconhecida.

Distribuição. Conhecido somente para a localidade-tipo. Material examinado: Somente os tipos.

Revista Brasileira de Zoologia 25 (1): 128-138, March, 2008

\section{Tenedos perfidus Jocqué \& Baert, 2002}

Tenedos perfidus Jocqué \& Baert, 2002: 112, figs 30 a-d, mapa 2; Platnick (2006).

Descrição. Ver Jocqué \& BAERT (2002).

Novos registros. BRAsiL, Tocantins: Palmas (Usina Hidrelétrica Luiz Eduardo Magalhães), 2 machos, 3 fêmeas, J. Guilher-

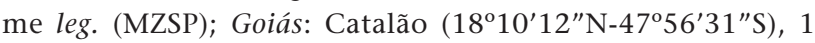
macho, 2002, P.C. Motta leg. (IBSP 38866); Mato Grosso: Cocalinho (Fazenda Globo), 1 macho, 1-10/XI/1997, M. Calleffo leg. (MZSP), Utiariti, 1 macho, 25/X/1966, Lenko \& Pereira leg. (MZSP); Minas Gerais: Uberlândia (Reserva de Caça e Pesca), 23 machos, 6 fêmeas, X/1996, D. Cunha leg. (IBSP 7810-7811; 7813; 7818-7819; 7821-7822; 7824).

\section{Tenedos procreator Jocqué \& Baert, 2002}

Tenedos procreator Jocqué \& Baert, 2002: 114, figs 32 a-e, mapa 2; Platnick (2006).

Descrição. Ver Jocqué \& BAERT (2002).

Novos registros. Brasil, São Paulo: Miracatú (Fazenda Itereí, $\left.24^{\circ} 03^{\prime} \mathrm{S}-47^{\circ} 13^{\prime} \mathrm{W}\right), 12$ machos, 20-26/IX/2001, Equipe Biota leg. (IBSP 53990, 45002); Paraná: Pinhão (Ribeirão Estreito, Usina Hidrelétrica Segredo), 1 fêmea, II/1992, R.P. da Rocha leg. (IBSP 54100); 2 fêmeas, II/1992, M. Segalla leg. (IBSP 54099); 1 macho, XI/1991, J.A.P. Barreto leg. (IBSP 54101).

\section{Tenedos infrarmatus Jocqué \& Baert, 2002}

Tenedos infrarmatus Jocqué \& Baert, 2002: 104, figs 22 a-b, mapa 1; Platnick (2006).

Descrição. Ver Jocqué \& BAERT (2002).

Novo registro. BRAsiL, Bahia: Gandú (Fazenda Pedra Branca), 1 macho, 5/II/1970, CEPLAC leg. (MNRJ).

\section{Tenedos hoeferi Jocqué \& Baert, 2002 Figs $40-48$}

Tenedos hoeferi Jocqué \& Baert, 2002: 99 figs 18a-e, mapa 1 (parátipos, Reserva Florestal Aldopho Ducke, Manaus, Amazonas, Brasil, H. Hoefer \& T. Gasnier leg., 1 macho, 18.XI.1991; 1 fêmea, 16.III.1991; INPA 0072 e INPA 0073, examinados); Platnick (2006).

Descrição. Ver Jocqué \& BAERT (2002). Acrescenta-se fotomicrografias com detalhes da morfologia do palpo do macho: ATR curta e romba; ATD de ápice agudo (Fig. 41); AM bem desenvolvida, bífida, com ponta distal em forma de gancho (Figs 40 e 42-45); região mediana e ápice do êmbolo com superfície porosa (Figs 46-48); êmbolo com abertura em forma de concha e ápice projetado lateralmente (Figs 47 e 48).

Variação. Espécimes de Senador Guiomard, Acre, apresentam AM com ponta proximal reduzida (Figs 42 e 43) ou ausente (Fig. 44); espécimes da Melgaço, Pará apresentam PTD afilado (Fig. 45).

Novos registros. Brasil, Amapá: Laranjal do Jarí (Cacho- 

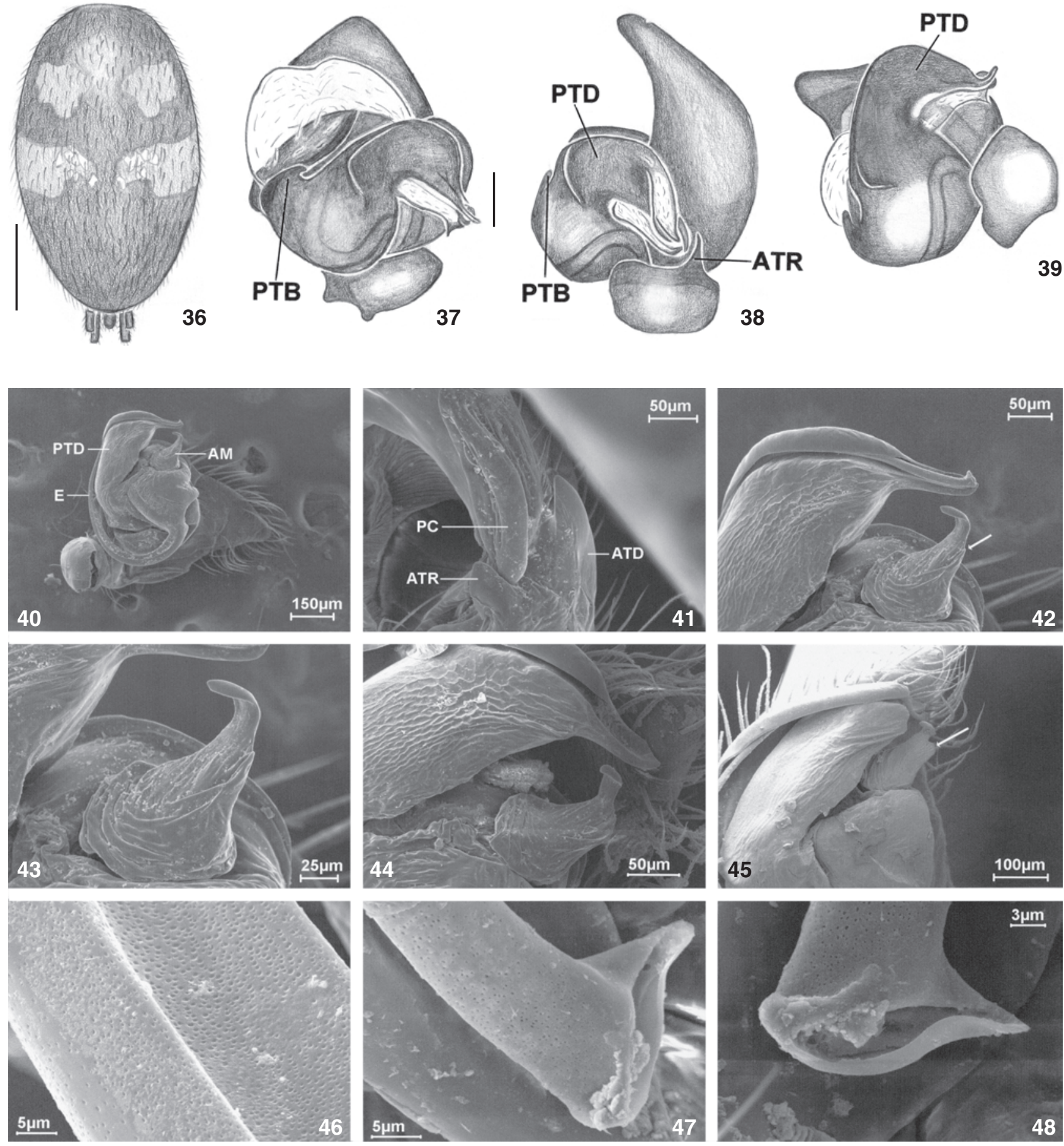

Figuras 36-48. (36-39) Tenedos carlosprestesi sp. nov., abdômen e palpo do macho: (36) abdômen dorsal; (37) palpo, ventral; (38) idem, retrolateral; (39) idem, retroventral. (ATR) Apófise tibial retrolateral, (PTB) processo tegular basal, (PTD) processo tegular distal. Barra: Fig. 36, 0,5 mm; Figs 37-39, 0,25 mm. (40-48) Tenedos hoeferi palpo do macho: (40) bulbo, ventral; (41) ápice da tíbia e base do címbio, retroventral; (42-45) ápice do bulbo, ventral; (46) base do êmbolo, ventral; (47-48) ápice do êmbolo, ventral. (AM) Apófise média, (PTD) processo tegular distal, (E) êmbolo, (ATR) apófise tibial retrolateral, (ATD) apófise tibial dorsal, (PC) processo cimbial, (Seta: AM) ponta proximal reduzida. Figuras 40-44, 46-48, Senador Guiomar, Acre; Fig. 45, Melgaço, Pará. 
eira de Santo Antonio), 1 macho, 2 fêmeas,19/II/2003, J.A.P. Barreiros leg. (MPEG 02301, 02303 e 02304); Pará: Monte Dourado (Almeirim, Reserva Genética do Pacanari), 1 fêmea, 20/II/ 2003, J.A.P. Barreiros leg. (MPEG 02302); Melgaço (Floresta Nacional de Caxiuanã, Estação Científica Ferreira Penna), 1 fêmea, (MPEG 02305); 8-16/VII/2002, 1 fêmea (MPEG 02306); 31/X-03/XI/2003, 1 macho (MPEG 02307); 11/VII/2002, 1 fêmea (MPEG 02308); 8-16/VII/2002, 2 fêmeas (MPEG 02311 e 02314); 21/III e 5-23/IV/2002, 2 machos, 3 fêmeas (MPEG 02324 e 02325); 2-5/XI/2002, 3 fêmeas (MPEG 02309, 02312 e 02313); 21-24/X/2003, 1 fêmea (MPEG 02310); 12/III/2003, 1 macho (MPEG 02315); 25/V/2003, 1 fêmea (MPEG 02316); 21/III/2003, 1 macho (MPEG 02317); 16/X/2003, 3 fêmeas (MPEG 02318 e 02319); 22/V/2003, 1 fêmea (MPEG 02320); 17/VIII/2003, 1 fêmea (MPEG 02321); 11/VIII/2003, 1 fêmea (MPEG 02322); 8/ VIII/2003, 2 fêmeas (MPEG 02323), 27-30/X/2003, todos coletados por J.A.P. Barreiros; Novo Progresso (Campo de Provas Brigadeiro Veloso, Serra do Cachimbo), 3 fêmeas (MPEG 02326, 03564, 03565); 7-17/IX/2003, 1 fêmea (MPEG 03572); 16-26/III/2004, 1 fêmea (MPEG 03573), 3-6/IV/2004, todos coletados por J. Ricetti; Acre: Senador Guiomard (Reserva extrativista de Catuaba), 12 machos, 2003, E. Morato leg. (IBSP 44537, 44538, 44540, 44541, 44542, 44543, 44544, 44545, 44546, 44547); Amazonas: Coari (Porto Urucu), 1 macho, 1/X/ 2004, A.B. Bonaldo et al. leg. (MPEG 02331); Manaus (Reserva Florestal Adolpho Ducke), 23/III/1992, H. Höfer leg., 3 machos, 1 fêmea (IBSP 10688, 10690, 10691 e 10695); 17/II/1994, M. Christofer leg., 2 machos (IBSP 54103 e 54104); 10/VIII/1992, M. Christofer leg., 1 fêmea (IBSP 54102).

\section{Epicratinus petropolitanus (Mello-Leitão, 1922) comb. nov.}

Storena petropolitana Mello-Leitão, 1922: 209 (Holótipo fêmea, Petrópolis, Rio de Janeiro, Brasil, Mello-Leitão leg., depositado no MNRJ 952, examinado por A. A. Lise); Platnick (2006).

\section{AGRADECIMENTOS}

A José A.M. Fernandes, Ana L. da C. Prudente (MPEG), Ricardo P. da Rocha (USP), Cristina Rheims (IBSP) e Nancy F.L.M. Hung (MPEG) pelas críticas e sugestões à versão acadêmica deste trabalho. A Arno A. Lise (PUC-RS) pela cessão das ilustrações de sua autoria dos tipos das espécies brasileiras descritas em Storena. A Rudy Jocqué (Musée Royal de L'Afrique Centrale) pelas informações sobre a identidade de zodarídeos neotropicais; a Hilton Costi, Laboratório de Microscopia Eletrônica (MPEG) pelo auxílio com as fotografias em microscopia eletrônica. Estudo apoiado pela CAPES (Bolsa de Mestrado, DFC), CNPq (Bolsas de Pesquisa, ABB, processo \#303591/2002-1 e ADB, processo \#301776/2004-0) e FAPESP (99/05446-8, ADB). Parte da Dissertação de Mestrado do primeiro autor, Curso de Pós-Graduação em Zoologia, Universidade Federal do Pará, Museu Paraense Emílio Goeldi, apoio BIOTA/FAPESP - "The Biodiversity Virtual Institute Program" (www.biotasp.org.br).

Revista Brasileira de Zoologia 25 (1): 128-138, March, 2008

\section{LITERATURA CITADA}

BAeHr, B. \& R. Jocqué. 1994. Phylogeny and zoogeography of the Australian genus Storena (Araneae, Zodariidae). Spixiana 17: $1-12$.

Bonaldo, A.B. 2000. Taxonomia da Subfamília Corinninae (Araneae, Corinnidae) nas Regiões Neotropical e Neártica. Iheringia, Série Zoologia 89: 3-148.

Chamberlin, R.V. 1925. Diagnoses of new American Arachnida. Bulletin of the Museum of Comparative Zoology 67: 209248.

Jocqué, R. 1991. A generic revision of the spider family Zodariidae (Araneae). Bulletin of American Museum of Natural History 201: 1-60.

JOCQUÉ, R. \& L. BAERT. 2002. A revision of the neotropical genera Tenedos O.P.-Cambridge and Ishania Chamberlin (Araneae, Zodariidae). Bulletin de L'institut Royal des Sciences Naturelles de Belgique, Entomologie 72: 67-173.

Jocqué, R. \& L. BAERT. 2005. Two new Neotropical genera of the spider family Zodariidae (Araneae). Bulletin de L'institut Royal des Sciences Naturelles de Belgique, Entomologie 75: 119-133.

Keyserling, E. 1891. Die Spinnen Amerikas. Brasilianische Spinnen. Nürnberg, Bauer, vol. 3, 278p.

Levi, H.W. 1965. Techniches for the study of spider genitalia. Psyche 72: 152-158.

Mello-Leitão, C.F. DE. 1922. Quelques Araignées Nouvelles ou peu Connues du Bresil. Annales de la Societé Entomologique de France 91: 209-228

Mello-Leitão, C.F. DE. 1925. Pequenas notas arachnologicas. Boletim do Museu Nacional do Rio de Janeiro 1: 455-463.

Mello-Leitão, C.F. DE. 1927. Arachnideos de Santa Catharina (Brasil). Revista do Museu Paulista 15: 393-418.

Mello-Leitão, C.F. DE. 1940. Arañas de la provincia de Buenos Aires y de las gobernaciones de La Pampa, Neuquén, Río Negro y Chubut. Revista del Museo de La Plata, Zoología, 2: 3-62.

Mello-Leitão, C.F. DE. 1941. Notes on Peruvian harvest-spiders. Anais da Academia Brasileira de Ciências 13: 319-322.

Petrunkevitch, A. 1911. A synonymic index-catalogue of spiders of North, Central and South America with all adjacent islands, Greeland, Bermuda, West Indies, Terra del Fuego, Galapagos, etc. Bulletin of American Museum of Natural History 29: $1-791$.

Pickard-Cambridge, O. 1897. Arachnida - Araneida, p. 225-232. In: R.H. PorTer (Ed.). Biologia Centrali-Americana. London, Zoology, I+317p.

Platnick, N.I. 2006. The world spider catalog, version 6.5. New York, American Museum of Natural History, Avalaible at: http://research.amnh.org/entomology/spiders/catalog/ index.html [Accessed in 24/VIII/2006].

Received in 01.XII.2006; accepted in 03.III.2008. 